\title{
Mild Traumatic Brain Injury Affects Cognitive Processing and Modifies Oscillatory Brain Activity during Attentional Tasks
}

\author{
Hanna Kaltiainen, ${ }^{1-3,5}$ Mia Liljeström, ${ }^{1,2}$ Liisa Helle, ${ }^{1,2,4}$ Anne Salo, ${ }^{5}$ Marja Hietanen, ${ }^{5}$ \\ Hanna Renvall, ${ }^{1,2,5,6}$ and Nina Forss ${ }^{1,2,5}$
}

\begin{abstract}
Despite the high prevalence of mild traumatic brain injury (mTBI), current diagnostic tools to objectively assess cognitive complaints after mTBI continue to be inadequate. Our aim was to identify neuronal correlates for cognitive difficulties in mTBI patients by evaluating the possible alterations in oscillatory brain activity during a behavioral task known to be sensitive to cognitive impairment after mTBI. We compared oscillatory brain activity during rest and cognitive tasks (Paced Auditory Serial Addition Test [PASAT] and a vigilance test [VT]) with magnetoencephalography between 25 mTBI patients and 20 healthy controls. Whereas VT induced no significant differences compared with resting state in either group, patients exhibited stronger attenuation of 8 - to $14-\mathrm{Hz}$ oscillatory activity during PASAT than healthy controls in the left parietotemporal cortex $(p \leq 0.05)$. Further, significant task-related modulation in the left superior frontal gyrus and right prefrontal cortex was detected only in patients. The $\sim 10-\mathrm{Hz}$ (alpha) peak frequency declined in frontal, temporal, and parietal regions during PASAT compared with rest $(p<0.016)$ in patients, whereas in controls it remained the same or showed a tendency to increase. In patients, the $\sim 10-\mathrm{Hz}$ peak amplitude was negatively correlated with behavioral performance in the Trail Making Test. The observed alterations in the cortical oscillatory activity during cognitive load may provide measurable neurophysiological correlates of cognitive difficulties in mTBI patients, even at the individual level.
\end{abstract}

Keywords: alpha frequency modulation; cognitive task; magnetoencephalography; mild traumatic brain injury

\section{Introduction}

$\mathbf{M}$ ILD TRAumatic BRAIN INJURIES (MTBI) are common: estimated 100-300 per 100,000 patients worldwide seek medical advice annually, and many others with mTBI do not contact any healthcare services. ${ }^{1,2}$ Although most mTBI patients show rapid and full recovery, some express long-lasting symptoms affecting their quality of life, ${ }^{3,4}$ and in the absence of objective trauma indicators, healthcare services may fail to recognize these patients. Residual symptoms after mTBI are usually minor, and after the subacute phase, performance in traditional neuropsychological test batteries is often within the normal range. ${ }^{3,5}$ Despite normal test performance, neuroimaging studies have suggested differences in neural processing after mTBI, lasting beyond the disappearance of clinical symptoms. ${ }^{6,7}$

Attentional deficits after mTBI are widely reported ${ }^{8,9}$ and suggested as the most sensitive indicator of cognitive dysfunction after mTBI, especially with simultaneous assessment of processing speed. ${ }^{10,11}$ Vigilance denotes maintaining an alert state in the surroundings with scarcity of external stimuli and may also be affected by mTBI. ${ }^{6,12,13}$ Vigilance can be probed with, for example, the sustained counting task in which the rate of pre-defined stimuli is slow and the task so easy that maintaining attention requires cognitive $\operatorname{control}^{14}$; increased activation in the right lateral frontal areas in healthy individuals has been suggested in this type of task. ${ }^{15}$

\footnotetext{
${ }^{1}$ Department of Neuroscience and Biomedical Engineering, Aalto University School of Science, Espoo, Finland.

${ }^{2}$ Aalto Neuroimaging, MEG Core, Aalto University, Espoo, Finland.

${ }^{3}$ Lohja District Hospital, Department of Neurology, Lohja, Finland.

${ }^{4}$ MEGIN (Elekta Oy), Helsinki, Finland.

${ }^{5}$ Clinical Neurosciences, University of Helsinki, and Department of Neurology, Helsinki University Hospital, ${ }^{6} \mathrm{HUS}$ Medical Imaging Center, BioMag Laboratory, University of Helsinki and Helsinki University Hospital, Helsinki, Finland.

(C) Hanna Kaltiainen et al., 2019; Published by Mary Ann Liebert, Inc. This Open Access article is distributed under the terms of the Creative Commons Attribution Noncommercial License (http://creativecommons.org/licenses/by-nc/4.0/) which permits any noncommercial use, distribution, and reproduction in any medium, provided the original author(s) and the source are credited.
} 
The Paced Auditory Serial Addition Test (PASAT) was originally developed for assessing changes that TBI causes in cognitive functioning, especially in speed of sensory information processing. ${ }^{16,17}$ Subsequently, the test has been demonstrated to probe multiple domains of cognitive functioning, such as sustained attention, concentration, and working memory. ${ }^{18-21}$ During the test, subjects listen to single-digit numbers and sum up every two consecutive numbers presented serially with a fixed interval. The test is typically presented sequentially with a declining interdigit interval, to make it more arduous. The test can be applied also in its visual form (PVSAT), but the auditory test has been considered especially sensitive for assessing TBI because it brings together two domains commonly affected in TBI: information processing speed and susceptibility to interference effects. ${ }^{21} \mathrm{mTBI}$ patients tested within $24 \mathrm{~h}$ after injury generally present lower scores compared to healthy controls, and improved test results correlate with diminishing clinical symptoms with repeated testing. ${ }^{16,22}$ Even chronically symptomatic mTBI patients, tested with PASAT months after injury, have performed significantly worse than healthy controls. ${ }^{16,23}$

In functional (fMRI) magnetic resonance (MR) imaging (MRI) studies of healthy subjects, the PASAT task activates middle frontal, as well as middle and superior temporal gyri with left predominance, together with bilateral inferior parietal lobules and anterior cingulate gyri. ${ }^{20,24-27}$ In repeated measurements (with a 6month interval), the activation tends to diminish, probably as a sign of learning. ${ }^{27}$ In some neurological patient groups, such as TBI and multiple sclerosis, cortical activation appears more widespread and right-lateralized than in controls, possibly attributed to recruitment of compensatory cortical areas during the task. ${ }^{20,28}$

The cerebral cortex has several intrinsic rhythms, of which the most prominent are the $\sim 10-\mathrm{Hz}$ alpha rhythm that can be recorded non-invasively over the parieto-occipital cortices with magnetoand electroencephalography (MEG/EEG). The alpha rhythm has been shown to modulate during attention-demanding working memory tasks, ${ }^{29,30-32}$ also outside the visual modality. ${ }^{29,30,33,34}$ The alpha rhythm typically attenuates in memory-related experimental paradigms in frontoparietal regions, ${ }^{35,36}$ and more powerful attenuation has correlated with better test performance. ${ }^{35,37}$ Occipitally, both alpha-frequency and power, however, seem to increase during attention-demanding tasks, ${ }^{31,34}$ and a tendency toward parieto-occipital alpha-frequency difference has been observed. ${ }^{31}$ Both alpha peak frequency ${ }^{38-40}$ and its task-induced modulations ${ }^{41,42}$ show good reproducibility over time in healthy individuals, thus making alpha oscillations a good candidate measure for evaluating possible changes during pathological cortical processes and after cortical trauma. Resting-state EEG early after TBI often presents a reduction in occipital alpha frequency, followed with a return to normal limits during subsequent months. ${ }^{43,44}$ Alpha peakfrequency decline has been associated with degraded white matter integrity, ${ }^{45}$ which is also compromised in mTBI patients suffering from traumatic axonal injury. ${ }^{46}$

Previously, we studied the appearance and modulation of resting-state low-frequency $(0.5-7.0 \mathrm{~Hz})$ oscillatory brain activity after mTBI, finding it often transient in repeated measurements, without clear correlation with resolution of self-reported symptoms. ${ }^{47}$ Here, our aim was to include in the MEG measurement protocol a cognitive task (PASAT) that is indicative of compromised cognitive processing in mTBI patients. ${ }^{16}$ Another, lessdemanding vigilance task and measurements during rest served as control conditions. We hypothesized that mTBI patients would show stronger attenuation of alpha power in frontoparietal cortices and more-widespread activation areas during PASAT com- pared to healthy controls, as a sign of increased attentional demands. Further, we hypothesized that stronger attenuation of alpha power would correlate with better neuropsychological performance of patients.

We hypothesized that in accord with earlier results with EEG ${ }^{43,44}$ patients would demonstrate a general shift in occipital alpha peak activity to higher frequencies along recovery. We also hypothesized that healthy controls would show more-prominent increase of occipital alpha frequency during PASAT, and thus divergent parieto-occipital alpha frequency modulation, during the attentional task compared to mTBI patients. Here, we utilized MEG for recording oscillatory brain activity: MEG permits estimation of cortical sources with good spatial accuracy, as well as assessment of oscillatory brain activity at the sensor level, an approach that is easily extendible to EEG recordings in a clinical environment. Within-group analyses are based on sensor-level data, where such comparisons are feasible. Because localization of alpha activity was of great interest here, we performed the between-groups analysis on alpha oscillatory power in source space, not at sensor level, where it is difficult to separate activity originating from close-by sources. To the best of our knowledge, this is the first study presenting cortical activation during PASAT with MEG.

\section{Methods}

\section{Subjects}

The patient group consisted of 25 TBI patients ( 11 females, 14 males) $42 \pm 2$ years of age (average \pm standard error of the mean [SEM]; females $44 \pm 3$, males $39 \pm 3$ ). Twelve patients underwent a single measurement within 6 months after injury, and 13 patients were measured at the subacute stage within 6 days to 2 months after injury; all but 1 participated in a follow-up MEG measurement at 6 months (Table 1). Nine of 12 with follow-up measurements participated also in a follow-up neuropsychological examination at $12.6 \pm 0.25$ (average \pm SEM) months after trauma. Trauma mechanisms included 10 bicycle accidents, six motor vehicle accidents, four falls, four sports-related accidents, and one hit to the head (Table 1).

The control group comprised 20 healthy adults ( 8 females, 12 males) $39 \pm 2$ years of age (females $44 \pm 3$, males $36 \pm 3$ ) without history of brain traumas. The Ethics Committee of Helsinki and Uusimaa Hospital District accepted the study.

\section{Clinical evaluation}

All patients and control subjects were without significant developmental or neuropsychiatric problems, medication affecting the central nervous system, substance abuse, or past TBIs. All patients fulfilled Glasgow Coma Scale (GCS) and duration of loss of consciousness (LOC) criteria for mTBI according to the American Congress of Rehabilitation Medicine ${ }^{48}$ and World Health Organization, ${ }^{49}$ that is, their GCS points were $\geq 13$ at 30 min after trauma and LOC was $<30 \mathrm{~min}$. In some patients, assessment of posttraumatic amnesia (PTA) was difficult because of concomitant injuries and use of sedatives and analgesics; therefore, it is possible that some patients had PTA for over $24 \mathrm{~h}$.

\section{Neuropsychological evaluation}

All patients filled in the Rivermead Post Concussion Symptom Questionnaire (RPQ) at the MEG measurement sessions and underwent careful neuropsychological testing at the subacute stage, $3.2 \pm 0.43$ (average \pm SEM) months after trauma. Neuropsychological 
Table 1. Demographics of the Patients ${ }^{\mathrm{a}}$

\begin{tabular}{|c|c|c|c|c|c|c|c|}
\hline Patient & Age & GCS & Type of trauma & MEG1 & $M E G 2$ & $R P Q 1$ & $R P Q 2$ \\
\hline 1 & 43 & 15 & bike accident & $4 \mathrm{~m}$ & & 3 & \\
\hline 2 & 50 & 15 & mva & $2 \mathrm{~m}$ & & 3 & \\
\hline 3 & 42 & 14 & sports accident & $5 \mathrm{~m}$ & & 24 & \\
\hline 4 & 46 & 14 & mva & $4.5 \mathrm{~m}$ & & 29 & \\
\hline 5 & 37 & 14 & bike accident & $3.5 \mathrm{~m}$ & & 13 & \\
\hline 6 & 32 & 15 & fall & $4 \mathrm{~m}$ & & 18 & \\
\hline 7 & 59 & 15 & bike accident & $3 w$ & & 3 & \\
\hline 8 & 54 & 15 & fall & $2 \mathrm{~m}$ & & 8 & \\
\hline 9 & 39 & 15 & mva & $2 \mathrm{~m}$ & & 31 & \\
\hline 10 & 20 & 14 & sports accident & $1 \mathrm{~m}$ & & 2 & \\
\hline 11 & 44 & 14 & mva & $1.5 \mathrm{~m}$ & & 27 & \\
\hline 12 & 43 & 14 & bike accident & $6 \mathrm{~m}$ & & 28 & \\
\hline 13 & 36 & 14 & mva & $1.5 \mathrm{~m}$ & & 25 & \\
\hline 14 & 39 & 15 & hit to head & $3 \mathrm{w}$ & $7 \mathrm{~m}$ & 9 & 7 \\
\hline 15 & 29 & 14 & sports accident & $1 \mathrm{~m}$ & $6 \mathrm{~m}$ & 3 & 2 \\
\hline 16 & 37 & 14 & mva & $1 \mathrm{~m}$ & $6 \mathrm{~m}$ & 25 & 14 \\
\hline 17 & 50 & 14 & fall & $2 \mathrm{~m}$ & $6 \mathrm{~m}$ & 6 & 3 \\
\hline 18 & 28 & 15 & bike accident & $1 \mathrm{w}$ & $6 \mathrm{~m}$ & 16 & 14 \\
\hline 19 & 29 & 14 & bike accident & $3 \mathrm{w}$ & $6 \mathrm{~m}$ & 3 & 2 \\
\hline 20 & 59 & 14 & bike accident & $1 \mathrm{w}$ & $6 \mathrm{~m}$ & 36 & 18 \\
\hline 21 & 53 & 14 & sports accident & $3 w$ & $6.5 \mathrm{~m}$ & 34 & 6 \\
\hline 22 & 51 & 15 & bike accident & $1 \mathrm{w}$ & $6 \mathrm{~m}$ & 14 & 6 \\
\hline 23 & 23 & 15 & bike accident & $1 \mathrm{w}$ & $6 \mathrm{~m}$ & 25 & 0 \\
\hline 24 & 40 & 14 & bike accident & $1 \mathrm{~m}$ & $7 \mathrm{~m}$ & 14 & 3 \\
\hline 25 & 56 & 15 & fall & $3 w$ & $6 \mathrm{~m}$ & 32 & 16 \\
\hline
\end{tabular}

${ }^{a}$ Age at the time of injury, GCS at 30 min after trauma, type of trauma, timing of magnetoencephalography (MEG) measurements (after injury), and RPQ scores at MEG measurements.

GCS, Glascow Coma Scale; mva, motor vehicle accident; m, months; w, weeks; RPQ, Rivermead Post-Concussion Symptom Questionnaire.

testing was implemented by two experienced neuropsychologists; Table 2 summarizes the tests included. Conversation, reading, writing, and counting skills were also evaluated. Digit Span, Trail Making Test A and B (TMA/B), Digit Symbol, and Stroop tests have earlier been shown to correlate with PASAT results in TBI patients, ${ }^{19,50}$ and they were here correlated with the MEG data. TMA and TMB tests measure processing speed and focused attention, ${ }^{21,50}$ and they, as well as the difference in time accomplishing those tests, were compared to the corresponding normative values in healthy controls.
In addition, patients and their significant others filled in The Dysexecutive (DEX) Questionnaire ${ }^{51}$ to rate difficulties in everydaylife executive functioning and the patients the Beck Depression Inventory (BDI) ${ }^{52}$ to evaluate concomitant mood changes.

The follow-up examination, in which 9 patients participated in, focused on executive functions, attention, processing speed, working memory, learning, and mood (utilizing Trail Making Test, Stroop, Fluency, Digit Span, List learning, Digit cancellation, BDI, and the DEX questionnaire).

Table 2. Neuropsychological Tests Administered

\begin{tabular}{lll}
\hline Neuropsychlogical test & \multicolumn{1}{c}{ Cognitive domain assessed } & Reference \\
\hline Wechsler Adult Intelligence Scale Third edition (WAIS-III) & Attention, working memory & Wechsler, 1997 \\
Digit Span, Letter Number & Reasoning, concept formation & \\
Similarities & General knowledge & \\
Information & Perceptual reasoning, organization & Wechsler, 1997b \\
Block Design & Reasoning, visual perception & \\
Picture Completion & Processing speed, attention & Benton, A. L., 1974 \\
Symbol Search, Digit-Symbol & & Reitan, 1958 \\
Wechsler Memory Scale (WMS-III) & Verbal memory & MacLeod, 1991 \\
Logical memory, List Learning & Visual memory & Burgess and Shallice, 1997 \\
Visual reproduction & Visual memory & Kaplan et al., 1983 \\
Benton Visual Retention Test & Attention, executive functions & Lezak et al., 2004 \\
Trail Making Test: A, B, difference & Attention, executive functions \\
Stroop Colour Naming Test & Executive functions & \\
Brixton Spatial Anticipation Test & Naming, verbal functions & \\
Boston Naming Test & Word fluency, executive functions &
\end{tabular}




\section{Magnetoencephalography and magnetic resonance imaging data collection}

We conducted the MEG measurements at Aalto Neuroimaging MEG Core (Aalto University, School of Science, Espoo, Finland) in a magnetically shielded room with a 306-channel whole-head device (Elekta Neuromag ${ }^{\mathrm{TM}}$; MEGIN [Elekta Oy], Helsinki, Finland), which comprises 102 triplet sensors with two planar gradiometers and one magnetometer coupled with a Superconducting Quantum Interference Device sensor. Before the measurement, digitized (Polhemus 3Space ${ }^{\circledR}$ Fastrak $^{\mathrm{TM}}$; Polhemus, Colchester, VT) nasion and two periauricular points determined the head coordinate system, and five head position indicator coils monitored the exact head position inside the helmet throughout the measurement session. Horizontal and vertical electro-oculogram and electrocardiogram provided data for artefact management. Collection of MEG signals occurred with a sampling rate of $1000 \mathrm{~Hz}$ and a band-pass filter of $0.03-330 \mathrm{~Hz}$.

The recordings comprised spontaneous resting-state activity measured with eyes open (EO) and eyes closed (EC), $10 \mathrm{~min}$ each, and during two cognitive tasks: the PASAT, ${ }^{16,17}$ and a vigilance test (VT). Measurements were performed in identical order (EO, EC, PASAT, and VT) in all patients and controls. After spontaneous activity recordings, subjects were allowed a short break, during which instructions for cognitive tests were repeated. For PASAT, we used 2.4-, 2.0-, and 1.6-sec presentation rates, and for those subjects (11 patients) who exhibited distress or made multiple mistakes during rehearsal at the 2.4-sec interval, we repeated the slower rate to exclude relinquishing effects during the test. The measurement during PASAT lasted for $6.5 \mathrm{~min}$. Subjects performed the test quietly without reporting the results aloud to avoid mouth movement artefacts. In VT, subjects listened to a story for $10 \mathrm{~min}$ and tried to count randomly scattered 13 words that were not part of the story. We used the first 5 min of EO, PASAT, and VT tasks for the analysis irrespective of the PASAT rate to obtain equal amount of averaged signal, with the task difficulty accommodated to gain maximal effort in both controls and patients.

During the tasks, subjects were asked to fixate on a fixation cross, sit relaxed, and avoid moving. During the EC condition, two, and during the EO condition one, short pauses confirmed that subjects remained alert.

All subjects underwent 1.5 Tesla anatomical MRI scans (GE Signa HDX 1.5 T; GE Healthcare, Milwaukee, WI), patients within 3 weeks to 16 months after the injury. Imaging consisted of sagittal $\mathrm{T}_{2}$ cube, fluid-attenuated inversion recovery, axial fast spin echo, axial three-dimensional (3D) susceptibility-weighted imaging, and axial 3D fast spoiled gradient-recalled echo $\mathrm{T}_{1}$ sequences.

\section{Data analysis}

Pre-processing. The temporal extension of the signal space separation method ${ }^{53}$ with movement compensation was utilized for artifact rejection. For residual eye-movement or cardiac artefacts, one component related to eye blinks or cardiac QRS-complex was projected out using independent component analysis. ${ }^{54}$ For sensorlevel analysis, we transferred all measurements to an average head position calculated over all patients and control subjects.

Sensor-level analysis. For data analysis, we applied relatively straightforward sensor-level peak power and frequency analyses, to facilitate later utilization of the methods in clinical settings and, possibly also, in EEG environment. Therefore, we focused on the alpha frequency range and sensor-level analysis of continuous MEG data.

We estimated sensor-level individual power spectra from the first 5 min of MEG data in each condition (EO, EC, PASAT, and VT). The power spectra were calculated using Welch's method with a 2048-point fast Fourier transform, 50\% overlap, and Hann windowing, resulting in a frequency resolution of $\sim 0.5 \mathrm{~Hz}$. We calculated areal averages for left and right frontal, parietal, temporal, and occipital sensor areas, to obtain areal peak frequencies and power at the alpha $(8-14 \mathrm{~Hz})$ frequency band. We concentrated on these frequencies and areas based on previous results on alpha band changes in healthy controls during cognitive tasks ${ }^{30,31,33}$ and during rest EEG recordings in mTBI patients after the injury. ${ }^{43,44}$ Alpha peak power and frequency were extracted for each participant as the peak values in the 8- to $14-\mathrm{Hz}$ frequency band over each sensor area and tested for within-group differences between conditions (PASAT vs. EO as well as VT vs. EO). Between-group comparisons of possible changes in alpha peak frequencies were performed at the sensor level, whereas between-group differences in power were estimated at the source level (see source modeling section).

Statistical analyses were conducted with IBM SPSS Statistics (version 24; IBM Corp., Armonk, NY) non-parametric tests, because of skewed distributions of the data. Frequency values were logarithmically transformed to enable comparisons. Mann-Whitney $\mathrm{U}$ tests served for between-groups analysis and Wilcoxon tests for within-groups analysis. Mixed analysis of variance (ANOVA; subject group as a between-groups factor, and task, area, and hemisphere as within-group factors) assessed between-groups interactions. Bonferroni correction for multiple comparisons was applied to Wilks' lambda results. When an interaction was observed, we used Student's two-tailed $t$-tests to further evaluate the values, ln-transformed to compensate for the unequal variances between groups. Correlations with the neuropsychological tests were calculated using Spearman's two-tailed rank-order correlation because of small sample size and skewed distributions of the data, and not corrected for multiple comparisons.

Source modeling. Source modeling of measured oscillatory brain activity was used to identify the cortical regions that contribute to the measured signals. Source modeling of MEG responses requires solving the so-called inverse problem, that is, estimating the origins of the oscillatory activity from the measurement data with the help of additional constraints. ${ }^{55}$ For MEG, origins of oscillatory activity can be estimated with up to 3-mm accuracy and sources located within a few centimeters from each other can be resolved. ${ }^{56,57}$ Because the source-modeling approach provides superior localization of the oscillatory power, we decided to perform between-groups power analysis at source space.

Source-level cortical activity was estimated using a spatial filter (or beamformer) in the frequency domain (dynamic imaging of coherent sources; DICS). ${ }^{57,58}$ DICS is a linearly constrained minimum variance beamformer that, given a cross-spectral density (CSD) matrix and a forward model of the neural currents, is designed to pass the activity in a specific location, while suppressing activity from other locations using a weighted sum of the sensor signals. ${ }^{59}$ The frequency-domain beamformer is well suited for identifying oscillatory activity in a continuous task. ${ }^{57,58}$ Data analysis was performed using MNE Python (v0.16; available at: https://martinos.org/mne/stable/auto_tutorials/plot_dics.html) ${ }^{60,61}$ and the ConPy python package (available at: https://github.com/ AaltoImagingLanguage/conpy $)^{62}$ with parameter choices as outlined in Van Vliet and colleagues. ${ }^{62}$ Within this approach, the pre- 
processed continuous planar gradiometer data, using the first $5 \mathrm{~min}$ of each condition to enable comparison between them, was first divided into 2.048-sec epochs. The CSD matrix was calculated to represent the covariance in the frequency domain between the signals at each sensor. To yield CSD matrices between all sensor combinations, time-frequency representations of epochs were calculated using Morlet wavelets with seven cycles. ${ }^{63}$ The obtained CSD matrices were subsequently averaged over all epochs and the entire time interval and the chosen 8 - to $14-\mathrm{Hz}$ frequency range.

A forward model, that is, a model that predicts the measured electromagnetic field generated by neural currents, is needed for computing the spatial filters. A single-layer boundary-element model, constructed from the inner skull surface of the MR images using FreeSurfer, ${ }^{64}$ was used as a head conductor model in the forward model. The possible neural sources were restricted to a surface-based cortical grid with 5124 nodes, established for each subject, thus allowing cortex-wide estimates of neural activity. $\mathrm{T}_{1}$ sequences of MR images, segmented using FreeSurfer, ${ }^{64}$ determined the individual cortical mantles. The neural currents at each grid point were modeled as equivalent current dipoles that represent the current as a vector with a magnitude and orientation. Spatial filters, constructed using the calculated CSD matrices and the forward model, selected the orientation with maximal power at each grid point. ${ }^{62}$ The resulting MEG power maps were morphed to an atlas brain (FS-average-5.1.0) to enable spatial comparison of the power maps across subjects and group-level statistical analysis.

Cluster-based permutation tests in MNE Python (corrected $p<0.05$ ), with a cluster-forming threshold of $p=0.00001$ for between-groups analysis and $p=0.01$ for within-group analysis, a maximal geodesic distance of 0.010 between adjacent vertices during clustering, and 10,000 permutations, assessed the potential statistical significance of differences in power between conditions and groups. Because of the individual variability of the brain oscillatory power, we normalized the data with each subject's maximum power in the resting-state EO condition for between-group comparisons. The Desikan-Killiany cortical parcellation atlas from FreeSurfer served for labeling the cortical areas. ${ }^{65}$

\section{Results}

\section{Sensor-level oscillatory brain activity}

Alpha peak power in controls and patients. Figure 1 presents the average areal spectra of oscillatory brain activity in controls and patients during rest and cognitive tasks. In both subject groups, PASAT induced attenuation of oscillatory activity (Fig. 1; Table 3). Control subjects showed statistically significant bilateral attenuation of alpha peak power in temporal, parietal, and frontal areas in the PASAT condition as compared with the EO condition (Fig. 1A; Table 3). Patients exhibited attenuation of alpha activity over the temporal and frontal cortices bilaterally and left parietal cortex, but not over the right parietal cortex (Fig. 1B; Table 3).

Modulation of oscillatory brain activity during the VT condition was modest, without significant differences compared with the EO condition (Fig. 1; Table 3).

Alpha peak frequency in controls and patients. A mixed ANOVA on the alpha frequencies revealed a significant interaction between cortical area and group $\left(F_{(1,43)}=6.91 ; p=0.01\right.$; partial $\left.\eta^{2}=0.14\right)$, as well as between cortical area and task $\left(F_{(2,42)}=4.66 ; p=0.02\right.$; partial $\eta^{2}=0.18$; Fig. 2$)$. At rest (EO), alpha peak frequency of controls did not differ between brain areas $(p>0.28)$, whereas in patients the alpha peak frequency was

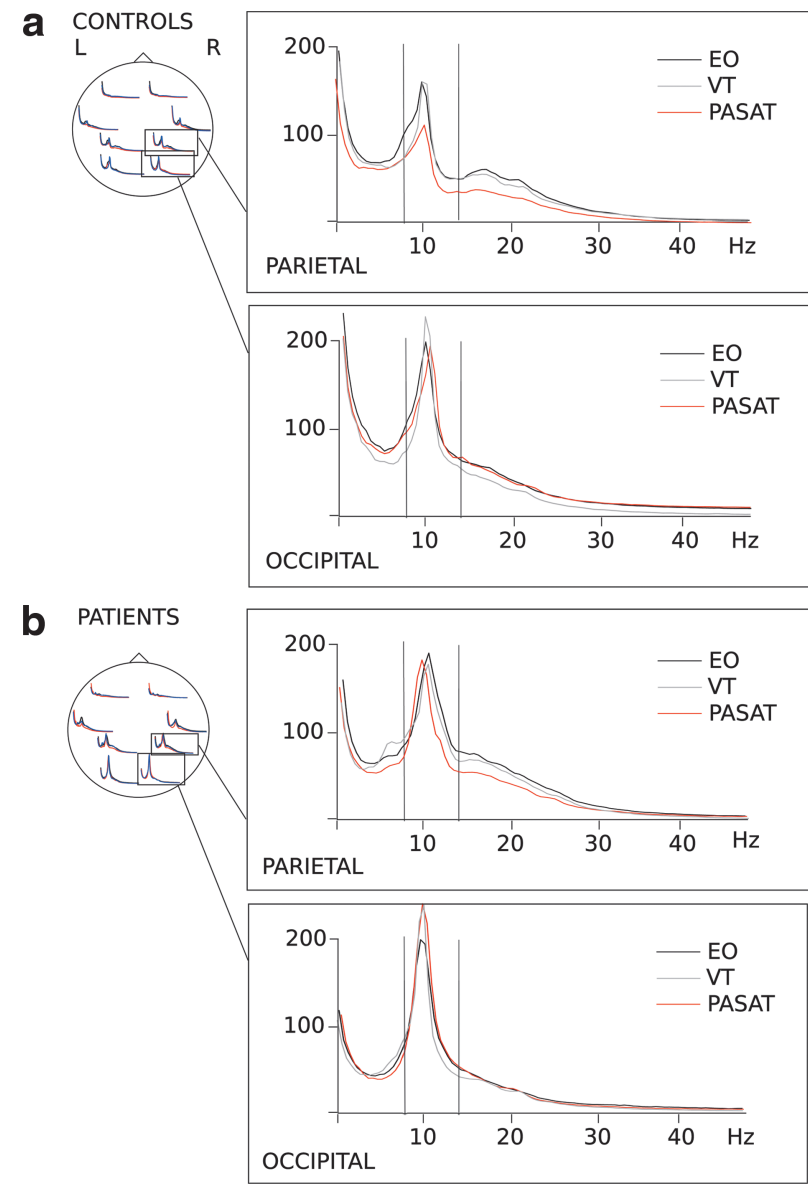

FIG. 1. Overview of the data. Sensor-level areal mean spectra for (A) control subjects and (B) patients. Inserts (right, A and B) show enlarged the mean spectra over the right parietal and occipital areas, demonstrating the activity during the tasks (VT, PASAT) compared with rest (EO). The vertical lines in the inserts mark the 8- to $14-\mathrm{Hz}$ band used for source-level power mapping. EO, eyes open; VT, vigilance test; PASAT, Paced Auditory Serial Addition Test; L, left; R, right.

significantly lower occipitally than parietally (right occipital 10.1 vs. parietal $10.7 \mathrm{~Hz}, \mathrm{Z}=-2.69, p=0.01$; left, 10.0 vs. $10.7 \mathrm{~Hz}$, $\mathrm{Z}=-2.55, p=0.02$; Fig. 2).

In controls, PASAT induced a frequency increase approaching significance in the left occipital area compared with EO (10.3 vs. $9.8 \mathrm{~Hz}, \mathrm{Z}=-1.89, p=0.06$; Fig. 2), whereas in other areas the difference between conditions was not significant $(p>0.11)$. Alpha frequencies were significantly higher in the right occipital $(10.5 \mathrm{~Hz})$ compared with the right parietal area during PASAT $(9.6 \mathrm{~Hz}$, $\mathrm{Z}=-2.29, p=0.03$; left, 10.3 vs. $9.7 \mathrm{~Hz}, \mathrm{Z}=-1.61, p=0.12$; Fig. 2 ).

In contrast, in patients PASAT induced slowing of the $\sim 10-\mathrm{Hz}$ activity in the parietal areas (left parietal, $10.7 \mathrm{~Hz}$ vs. $9.9 \mathrm{~Hz}$, $\mathrm{Z}=-2.52, p=0.01$; Fig. 2$)$, but not in the occipital areas $(p>0.6)$. During PASAT, the alpha peak frequencies did not differ significantly between the cortical areas (Fig. 2).

Between groups, the only significant difference resided in the left parietal region, where the alpha frequency of patients in EO condition was higher than in controls (patients, $10.7 \mathrm{~Hz}$ vs. controls, $9.6 \mathrm{~Hz} ; \mathrm{Z}=-2.67, p=0.01$ ).

VT induced no significant differences in alpha frequencies in either group compared with rest. 
Table 3. Channel-Level Alpha Peak Power Changes Between Rest and Activations

\begin{tabular}{|c|c|c|c|c|c|c|c|c|}
\hline \multirow[b]{2}{*}{ Area } & \multicolumn{4}{|c|}{ Controls } & \multicolumn{4}{|c|}{ Patients } \\
\hline & $Z(E O / P)$ & $\mathrm{p}(E O / P)$ & $Z(E O / V)$ & $\mathrm{p}(E O / V)$ & $Z(E O / P)$ & $\mathrm{p}(E O / P)$ & $Z(E O / V)$ & $\mathrm{p}(E O / V)$ \\
\hline LF & -3.136 & 0.002 & -0.523 & 0.601 & -2.785 & 0.005 & -1.117 & 0.264 \\
\hline $\mathrm{RF}$ & -3.024 & 0.002 & -0.187 & 0.852 & -3.108 & 0.002 & -1.958 & 0.056 \\
\hline $\mathrm{LT}$ & -3.061 & 0.002 & -1.157 & 0.247 & -3.834 & $<0.001$ & -1.843 & 0.065 \\
\hline RT & -2.501 & 0.012 & -0.896 & 0.370 & -2.408 & 0.016 & -1.144 & 0.253 \\
\hline LP & -2.427 & 0.015 & -1.195 & 0.232 & -2.085 & 0.037 & -0.013 & 0.989 \\
\hline $\mathrm{RP}$ & -2.315 & 0.021 & -0.448 & 0.654 & -1.305 & 0.192 & -0.175 & 0.861 \\
\hline LO & -1.493 & 0.135 & -0.672 & 0.502 & -0.821 & 0.412 & -0.955 & 0.339 \\
\hline RO & -0.709 & 0.478 & -0.485 & 0.627 & -0.013 & 0.989 & -1.547 & 0.122 \\
\hline
\end{tabular}

The bolded values indicate statistically significant results.

LF, left frontal; RF, right frontal; LT, left temporal; RT, right temporal; LP, left parietal; RP, right parietal; LO, left occipital; RO, right occipital; EO, eyes open; P, Paced Auditory Serial Addition Test; V, vigilance test.

\section{Source-level oscillatory brain activity}

Figure 3 and Table 4 depict results from the source-level analysis. In line with the sensor-level analysis (Fig. 1), control subjects (Fig. 3A) displayed significant $(p<0.05$, corrected) attenuation of oscillatory power in the 8 - to $14-\mathrm{Hz}$ band in the bilateral superior temporal gyri and sulci, left middle and inferior temporal, parahippocampal and fusiform gyri, bilateral pre-central and post-central cortices, bilateral supramarginal gyri, and inferior parietal cortices during PASAT compared with the EO condition. Similarly to controls, the patients (Fig. 3B; Table 4) exhibited attenuation ( $p<0.05$, corrected) bilaterally in the frontotemporal and parietal areas; additionally, in patients, oscillatory power was statistically significantly attenuated in the superior and rostral middle frontal gyri, left caudal middle frontal gyrus, bilateral pars opercularis and paracentral lobules, left cingulate cortex, right middle and inferior temporal gyri, left entorhinal cortex, and temporal pole, but not in left banks superior temporal sulcus.

Between-group analysis revealed statistically significantly stronger 8- to $14-\mathrm{Hz}$ oscillatory power attenuation during PASAT in patients than in controls ( $p<0.05$, corrected) in the bilateral frontal and parietal cortices (Table 4 ). These areas include supramarginal gyrus, angular gyrus, dorsolateral pre-frontal cortex (DLPFC), dorsomedial prefrontal cortex (DMPFC), and pre-central and paracentral areas (Fig. 3C).

In the VT condition, oscillatory brain activity did not significantly differ compared with rest in either group or between groups.

\section{Follow-up measurements}

Source space analysis of the 12 patients with control measurements did not indicate significant changes between the two

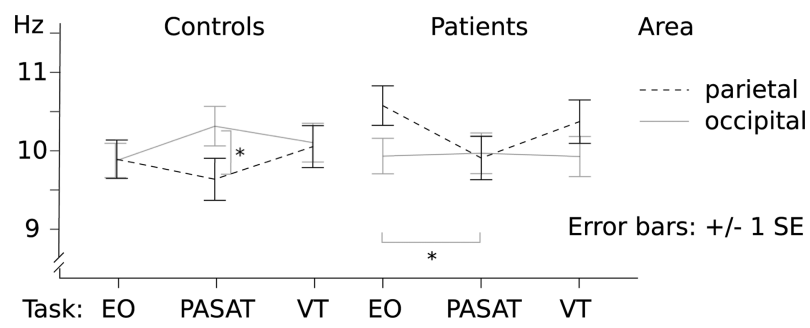

FIG. 2. Alpha peak frequency modulation during the cognitive tasks (PASAT, VT) compared with rest (EO) in parietal and occipital areas, both hemispheres unified. Asterisks denote statistically significant difference: in controls between parietal and occipital areas during PASAT, and in patients between EO and PASAT tasks in parietal areas. EO, eyes open; PASAT, Paced Auditory Serial Addition Test; VT, vigilance test; SE, standard error. measurement sessions. In sensor-level analysis, neither the alpha peak frequencies $(p>0.2)$ nor the peak powers $(p>0.1)$ differed between the two measurement sessions during the cognitive tasks. Occipital alpha peak frequencies remained stable between the two measurement sessions during rest (EO) and cognitive tasks $(p>0.08)$.

The second measurement session indicated no significant interaction between cortical area and task (PASAT, VT, and EO), although a significant main effect of area $(p=0.02)$ was observed. During the second measurement, PASAT induced slowing of alpha peak frequencies compared with EO in the right parietal area (11.4 vs. $10.0 \mathrm{~Hz} ; \mathrm{Z}=-2.25 ; p=0.02)$, similarly to the first measurement. Also in line with the first measurements, the alpha peak frequency at rest was significantly lower occipitally than parietally (left occipital, $10.1 \mathrm{~Hz}$ vs. parietal, $11.6 \mathrm{~Hz}, \mathrm{Z}=-2.32, p=0.02$; right occipital, $10.5 \mathrm{~Hz}$ vs. parietal, $10.4 \mathrm{~Hz}, \mathrm{Z}=-1.61, p=0.11$ ), whereas no frequency variation was observed during the cognitive tasks (PASAT, VT; $p>0.1$ ).

\section{Neuropsychological findings}

Residual neuropsychological symptoms of the patients at the subacute stage, $3.2 \pm 0.43$ (average \pm SEM) months after trauma, were mild. The most frequent residual symptoms patients reported were fatigue (84\%) and increased irritability (64\%). According to the BDI, $32 \%$ of patients obtained scores indicating depression; in $20 \%$ of patients, these symptoms were mild and in $12 \%$ mild to moderate. Neuropsychological testing revealed minor problems of working memory (in $64 \%$ of the patients), attention (36\%), flexibility, initiative or processing speed (32\%), planning (28\%), and abstract thinking or inhibition (24\%).

Behaviorally, the simple sustained counting task administered during VT revealed a significant difference between patients and controls: 18 of 20 controls reported \pm 1 correct result, whereas only 15 of 25 patients did so in the first measurement session (chi-square statistic, $5.114 ; p<0.02$ ). Of the patients with follow-up measurements, 9 of 12 (first measurement) and 10 of 12 (second measurement) reported \pm 1 correct result; the difference between controls and this subgroup was not significant.

\section{Correlating neuropsychological findings with magnetoencephalography data}

We compared the relevant neuropsychological tests (see Methods: Neuropsychological evaluation) with the statistically significant alpha power effects observed at the sensor level in 25 patients 
a Controls: PASAT - EO
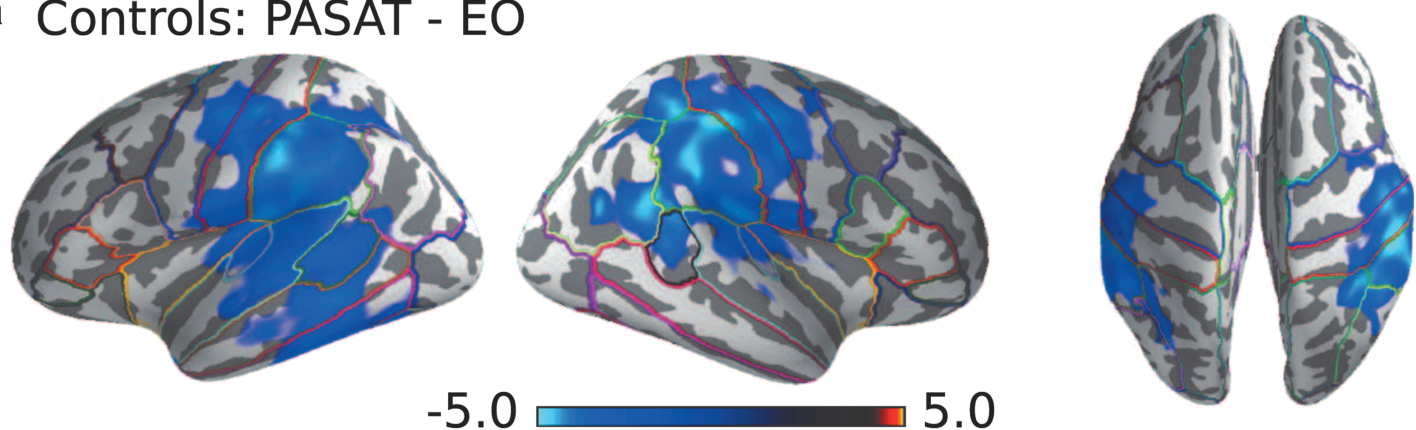

b Patients: PASAT -EO
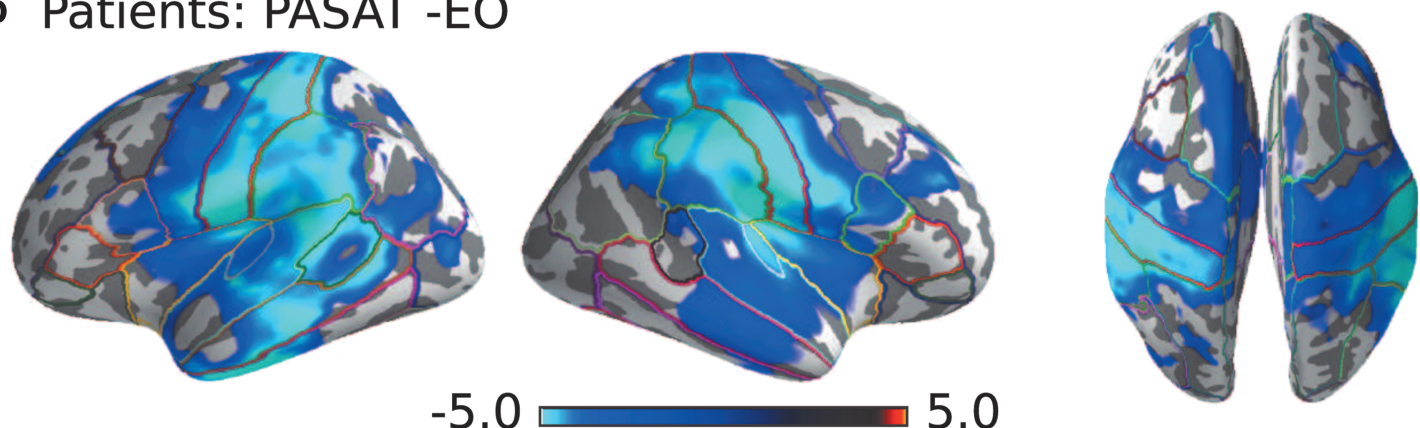

\section{c PASAT: Patients - Controls}
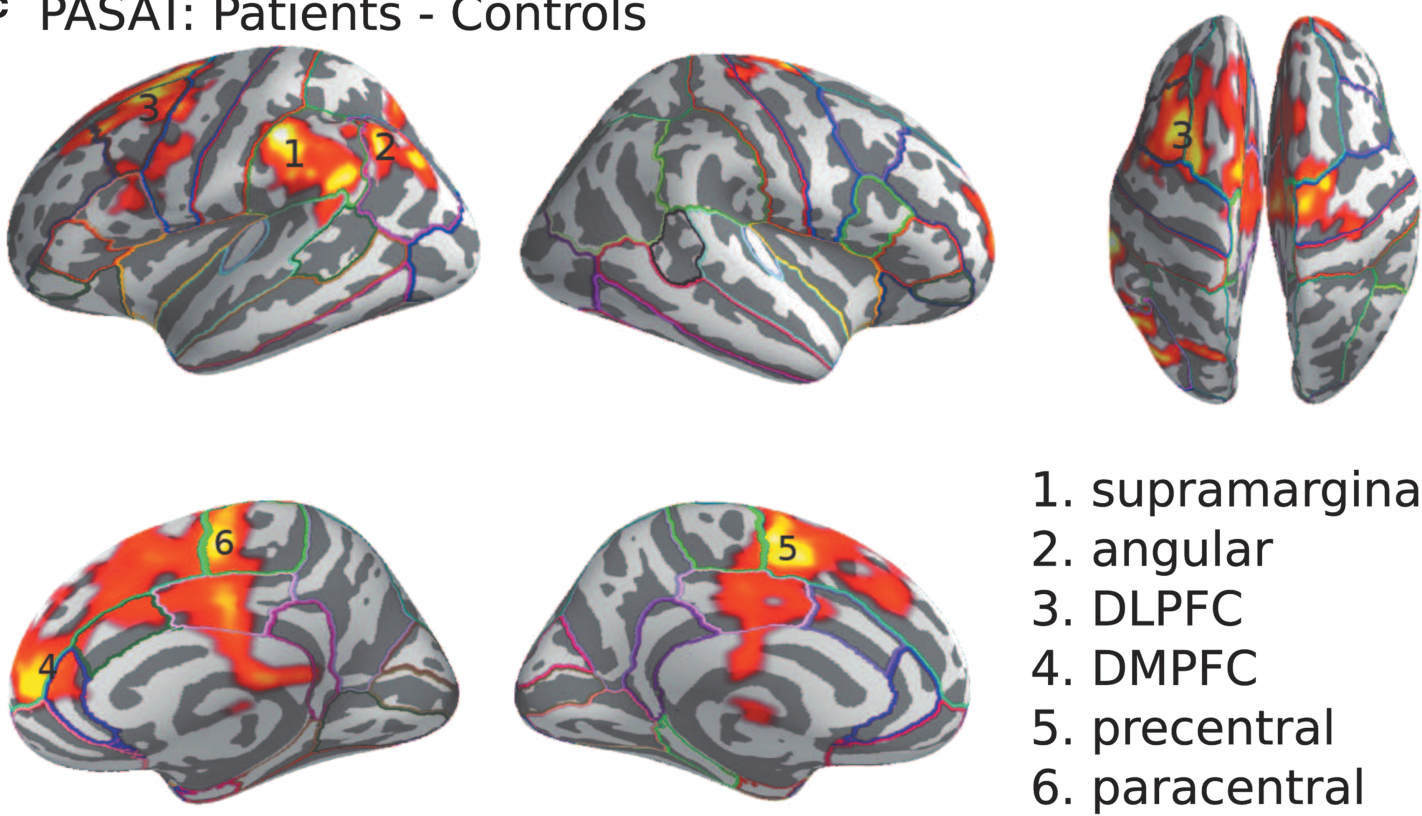

1. supramarginal

2. angular

3. DLPFC

4. DMPFC

5. precentral

6. paracentral

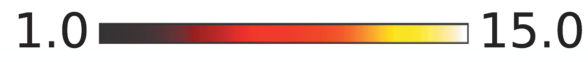

FIG. 3. Activation patterns during the PASAT. (A) Controls exhibit 8 - to $14-\mathrm{Hz}$ attenuation $(p<0.05$, corrected) in the bilateral fronto-parieto-temporal areas during the PASAT compared with EO, whereas (B) patients show statically significant attenuation $(p<0.05$, corrected) also in the dorsolateral and -medial pre-frontal cortices. (C) Between-group comparison during PASAT depicts stronger attenuation of 8 - to $14-\mathrm{Hz}$ power $(p<0.05$, corrected) in patients than controls in the left supramarginal and angular gyri, left DLPFC, right DMPFC, bilateral precentral, and left paracentral areas. PASAT, Paced Auditory Serial Addition Test; VT, vigilance test; EO, eyes open; DLPFC, dorsolateral pre-frontal cortex; DMPFC, dorsomedial pre-frontal cortex.

during the first PASAT measurement session. The low number of patients participating in the follow-up measurement (12) and neuropsychological assessment (9) prevented correlation analysis of the follow-up data. We found a correlation with the power results and TMB/TMA difference; in TMA, the subject connects 25 con- secutive numbers with a pen, and in TMB 12 consecutive numbers and 12 first alphabets alternating between numbers and letters, as swiftly as possible. The time difference in completing these two tasks was transformed to percentiles of healthy individuals' performance. The right temporal $(\mathrm{k}=-0.72 ; p=0.003)$ and left parietal 
Table 4. Activation Areas during PaSAT Compared with Rest

\begin{tabular}{|c|c|c|c|}
\hline Area & Controls & Patients & $c / p$ difference \\
\hline Superior frontal gyrus BA 6, BA 8-9 & & $\mathrm{L}+\mathrm{R}$ & $\mathrm{L}+\mathrm{R}$ \\
\hline Rostral middle frontal gyrus BA 46, BA 8-9 & & $\mathrm{L}+\mathrm{R}$ & $\mathrm{L}+\mathrm{R}$ \\
\hline Caudal middle frontal gyrus BA 6 & & $\mathrm{~L}$ & $\mathrm{~L}$ \\
\hline Pars opercularis BA 44 & & $\mathrm{~L}+\mathrm{R}$ & $\mathrm{L}$ \\
\hline Pre-central gyrus BA 4 & $\mathrm{~L}+\mathrm{R}$ & $\mathrm{L}+\mathrm{R}$ & \\
\hline Paracentral lobule BA $1-3$ & & $\mathrm{~L}+\mathrm{R}$ & $\mathrm{L}+\mathrm{R}$ \\
\hline Caudal anterior-cingulate cortex BA 24, BA 32 & & $\mathrm{~L}$ & $\mathrm{~L}+\mathrm{R}$ \\
\hline Posterior-cingulate cortex BA $23, \mathrm{BA} 31$ & & $\mathrm{~L}$ & $\mathrm{~L}+\mathrm{R}$ \\
\hline Post-central gyrus BA $1-3$ & $\mathrm{~L}+\mathrm{R}$ & $\mathrm{L}+\mathrm{R}$ & \\
\hline Supramarginal gyrus BA 40 & $\mathrm{~L}+\mathrm{R}$ & $\mathrm{L}+\mathrm{R}$ & $\mathrm{L}$ \\
\hline Superior parietal cortex BA 5 & $\mathrm{~L}+\mathrm{R}$ & $\mathrm{L}+\mathrm{R}$ & $\mathrm{L}$ \\
\hline Inferior parietal cortex BA 39 & $\mathrm{~L}+\mathrm{R}$ & $\mathrm{L}+\mathrm{R}$ & $\mathrm{L}$ \\
\hline Superior temporal gyrus BA 22 & $\mathrm{~L}+\mathrm{R}$ & $\mathrm{L}+\mathrm{R}$ & $\mathrm{L}$ \\
\hline Middle temporal gyrus BA 21 & $\mathrm{~L}$ & $\mathrm{~L}+\mathrm{R}$ & \\
\hline Inferior temporal gyrus BA 20 & $\mathrm{~L}$ & $\mathrm{~L}+\mathrm{R}$ & \\
\hline Banks superior temporal sulcus BA 37 & $\mathrm{~L}+\mathrm{R}$ & $\mathrm{L}$ & \\
\hline Entorhinal cortex BA 28 & & $\mathrm{~L}$ & \\
\hline Parahippocampal gyrus BA 36 & $\mathrm{~L}$ & $\mathrm{~L}$ & \\
\hline Temporal pole BA 38 & & $\mathrm{~L}$ & \\
\hline Fusiform gyrus BA 37 & $\mathrm{~L}$ & $\mathrm{~L}$ & \\
\hline
\end{tabular}

PASAT, Paced AuditorySerial Addition Test; BA, Brodmann areas; L, left; R, right; difference, areas with different activation patterns in betweengroups comparison.

$(\mathrm{k}=-0.64 ; p=0.01)$ peak powers within the alpha band correlated negatively with the TMA and TMB difference. Lower right temporal and left parietal peak powers were thus associated with better TM performance in patients.

\section{Discussion}

\section{Modulation of alpha peak frequencies during Paced Auditory Serial Addition Test}

Our study demonstrates alterations in oscillatory activity during PASAT in mTBI patients using continuous MEG measurements. In addition to source localization of neural oscillatory activity, we wanted to implement sensor-level analysis for easier transition of analysis pipeline to clinical use with EEG. The parieto-occipital alpha peak frequency differed between PASAT and rest in both patients and controls, but in a qualitatively different manner. In controls, the peak frequencies did not change in any other area, but appeared to increase occipitally during PASAT, whereas in patients the peak frequencies declined significantly in the left parietal area, but remained stable occipitally (Fig. 2).

In healthy subjects, increase of alpha peak frequencies has earlier been demonstrated during highly demanding cognitive processing ${ }^{31,66}$ as well as after strenuous exercise, ${ }^{67}$ and it has been associated with the activation level of neuronal populations. ${ }^{68}$ Compatible with our results, Haegens and colleagues found an increase in alpha peak frequency specifically in the posterior cortical regions during demanding cognitive tasks in healthy subjects. ${ }^{31}$ Therefore, our observation of a decrease of alpha peak frequencies over the parietal cortical area during a stressful cognitive task in $\mathrm{mTBI}$ patients is a surprising finding and an important indicator of altered information processing. The decline in alpha peak frequency may result from aforementioned white matter damage,${ }^{45}$ but it has also been associated with different functional states, such as meditation, ${ }^{69}$ and certain emotions, such as fear and sorrow. ${ }^{70}$ In this setting, the alpha frequency decrease in the parietal area observed in mTBI patients, together with the failure to increase occipital alpha frequency, might reflect axonal injury affecting white matter integrity. This, in turn, leads to reduced capacity in the interplay between distinct brain areas needed for the high-level performance during PASAT, a task demanding integrated activity of multiple cognitive domains.

\section{Modulation of oscillatory power during Paced Auditory Serial Addition Test}

Both control subjects and patients presented significant attenuation of cortical oscillatory activity in the temporo-parieto-frontal areas during PASAT compared with rest. Our results are in line with the previous fMRI and positron emission tomography studies of PASAT task in healthy subjects observing neural activation in compatible cortical areas than reported here. ${ }^{24-26}$ Equally with our results, a study with EEG-evoked responses ${ }^{71}$ have indicated significant activation differences in multiple frontal areas in patients compared to healthy subjects. Here, the source space analysis of the underlying cortical activation patterns showed that the attenuation of oscillatory power in patients was significantly stronger compared to controls in the left supramarginal and angular gyri, which form the inferior parietal lobule (Fig. 3C). Previous studies have shown that a lesion within this area, ${ }^{72,73}$ or disruption of neural activity within this region by repetitive transcranial magnetic stimulation, ${ }^{74}$ results in impaired addition capability.

Patients displayed stronger attenuation of oscillatory activity also in the right dorsomedial (DMPFC) and dorsolateral (DLPFC) pre-frontal cortices. DLPFC is important in monitoring and manipulation of working memory substance, ${ }^{75,76}$ and the right DLPFC has been associated with arithmetic functions. ${ }^{77}$ DMPFC has a role in maintenance of attention and motivation. ${ }^{75,76}$ Working memory paradigms have often activated large areas, including pre-motor and parietal cortices, ${ }^{76}$ similarly to our study. According to previous studies with fMRI, TBI and multiple sclerosis patients tend to activate larger and multiple areas compared to controls during the PASAT, ${ }^{20,28}$ which was also true for our mTBI patients using the MEG method. Here, activated areas in patients were quite 
symmetrical, without left lateralization of temporal activation probably related to the auditory processing in earlier fMRI studies. Specifically, patients seemed to activate multiple frontal areas compared to healthy controls (Fig. 3C; Table 4). Our findings thus suggest an enhanced frontal control in mTBI patients during the PASAT, similarly to an earlier EEG study with event-related potentials. $^{7}$

\section{Correlation of behavioral tests with measured oscillatory activity}

Importantly, patients' performance in the Trail Making Test correlated with peak amplitude values over the right temporal and left parietal cortices during the PASAT. This finding is in line with previous literature on healthy participants, where stronger alphaband attenuation during working memory tasks has been associated with successful memory performance. ${ }^{35,78}$ The differences in activation patterns between patients and controls, that is, the more intensive attenuation of oscillatory power and involvement of frontal cortices during the PASAT in patients, might thus relate to the inefficient cognitive functioning in these patients, resulting in enhanced need for task execution monitoring to compensate trauma-induced deficiency.

During the less-demanding VT condition, significant alterations in oscillatory activity compared with rest were not observed in either subject group. This may reflect the smaller cognitive load of the task, and the result also confirms that the observed differences in oscillatory activity during the PASAT compared with rest were not attributed to general differences in attention between groups. The behavioral results during VT, however, differed significantly between control and patient groups, connoting a difference in neural processing.

\section{Oscillatory activity in repeated measurements}

In our 12 patients with repeated measurements at 6 months, the observed task-related changes in oscillatory activity did not alter significantly, suggesting that the observed neurophysiological effects may be rather long-lasting and thus observable also later in time. In the earlier studies presenting significant increase in occipital alpha frequency during recovery, the first measurement session was conducted within $48 \mathrm{~h}$, whereas we were able to perform the first measurement at earliest 6 days after injury, possibly explaining part of the difference between the earlier and our results. ${ }^{43,44}$ In our previous study assessing low-frequency oscillatory activity, the observed aberrant activity disappeared in 3 of 5 patients by the second measurement at 6 months. ${ }^{47}$ Therefore, adding a strenuous cognitive task such as the PASAT to the measurement protocol might offer additional information in mTBI patients with cognitive complaints also later during the follow-up. However, the small number of patients attending the follow-up neuropsychological evaluation prevents attempting correlation of these oscillatory changes with the behavioral test results.

Failure of the conventional clinical evaluation methods to identify patients with compromised cognitive resources validates the need for future research. In our study, mTBI patients presented a decline in areal alpha peak frequencies within activated areas during a cognitive task, compared with rest. Patients also exhibited areal alpha peak frequency difference at rest, with lower occipital alpha peak frequency compared with the parietal region. These findings might offer new indicators for inefficient cognitive processing, easily assessed in individual patients using continuous EEG recordings during rest and demanding cognitive tasks such as the PASAT. Future studies should thus confirm the modulation of alpha peak frequency during the PASAT in a large database of healthy subjects and, subsequently, aim at individual patient level using preferably EEG because of its easier availability in clinical practice. The deviant modulation of areal alpha oscillatory activity in patients during the PASAT might open possibilities for continuous EEG monitoring during cognitive testing, an approach already utilized in aviation for assessing cognitive load in air traffic controllers. ${ }^{79}$

\section{Conclusions}

During a challenging cognitive task PASAT, mTBI patients exhibit stronger attenuation of 8 - to $14-\mathrm{Hz}$ power in task-relevant cortical regions in the inferior parietal lobule and frontal cortex compared to healthy controls, suggesting need for enhanced neural processing and frontal cognitive control after mTBI; the effects observed in the MEG measurements correlated with relevant behavioral test results. Further, given that areal alpha frequency modulation during the PASAT differentiated mTBI patients from healthy controls, the finding might serve as a sensitive indicator of cognitive problems also in clinical use. Future research is needed to confirm this pattern, and to develop further ways of assessing single patients in clinical environments.

\section{Acknowledgments}

This research has been funded by TEKES, the Finnish Funding for Innovation, Seamless patient care project funding (H.K., N.F.), State Research funding grant number 1988/31/2015 (H.K., N.F.), The Paulo Foundation (H.K.), The Finnish Medical Society Duodecim (H.K.), Academy of Finland grant number 277655 (H.R.), the Finnish Cultural Foundation (H.R.), Maud Kuistila Memorial Foundation (M.L.), and The Swedish cultural foundation (M.L.).

We cordially thank Ms. Mia Illman for help with MEG measurements, Jussi Numminen, MD, PhD, for providing MRI reports, and Psl Kaisa Mäki for help with neuropsychological testing of the patients.

\section{Author Disclosure Statement}

Author Liisa Helle is employed by a commercial company MEGIN (Elekta Oy), which designs, manufactures, and markets MEG devices. For all the other authors, no competing financial interests exist.

\section{References}

1. Cassidy, J.D., Carroll, L.J., Peloso, P.M., Borg, J., von Holst, H., Holm, L., Kraus, J., and Coronado, V.G.; WHO Collaborating Centre Task Force on Mild Traumatic Brain Injury. (2004). Incidence, risk factors and prevention of mild traumatic brain injury: results of the WHO Collaborating Centre Task Force on Mild Traumatic Brain Injury. J Rehabil Med. 43, Suppl., 28-60.

2. Gardner, R.C., and Yaffe, K. (2015). Epidemiology of mild traumatic brain injury and neurodegenerative disease. Mol. Cell. Neurosci. 66, 75-80.

3. Dikmen, S., Machamer, J., and Temkin, N. (2017). Mild traumatic brain injury: longitudinal study of cognition, functional status, and post-traumatic symptoms. J. Neurotrauma 34, 1524-1530.

4. Waljas, M., Iverson, G.L., Lange, R.T., Hakulinen, U., Dastidar, P., Huhtala, H., Liimatainen, S., Hartikainen, K., and Ohman, J. (2015). A prospective biopsychosocial study of the persistent post-concussion symptoms following mild traumatic brain injury. J. Neurotrauma 32, 534-547.

5. Pontifex, M.B., Broglio, S.P., Drollette, E.S., Scudder, M.R., Johnson, C.R., O'Connor, P.M., and Hillman, C.H. (2012). The relation of mild 
traumatic brain injury to chronic lapses of attention. Res. Q. Exerc. Sport 83, 553-559.

6. Pontifex, M.B., O'Connor, P.M., Broglio, S.P., and Hillman, C.H. (2009). The association between mild traumatic brain injury history and cognitive control. Neuropsychologia 47, 3210-3216.

7. Rogers, J.M., Fox, A.M., and Donnelly, J. (2015). Impaired practice effects following mild traumatic brain injury: an event-related potential investigation. Brain Inj. 29, 343-351.

8. Binder, L.M., Rohling, M.L., and Larrabee, G.J. (1997). A review of mild head trauma. part I: meta-analytic review of neuropsychological studies. J. Clin. Exp. Neuropsychol. 19, 421-431.

9. Barker-Collo, S., Jones, K., Theadom, A., Starkey, N., Dowell, A., McPherson, K., Ameratunga, S., Dudley, M., Te Ao, B., and Feigin, V.; BIONIC Research Group. (2015). Neuropsychological outcome and its correlates in the first year after adult mild traumatic brain injury: a population-based New Zealand study. Brain Inj. 29, 16041616.

10. Cicerone, K.D., and Azulay, J. (2002). Diagnostic utility of attention measures in postconcussion syndrome. Clin. Neuropsychol. 16, 280-289.

11. Frencham, K.A., Fox, A.M., and Maybery, M.T. (2005). Neuropsychological studies of mild traumatic brain injury: a metaanalytic review of research since 1995. J. Clin. Exp. Neuropsychol. 27, 334-351.

12. Burg, J.S., Burright, R.G., and Donovick, P.J. (1995). Performance data for traumatic brain-injured subjects on the Gordon Diagnostic System (GDS) tests of attention. Brain Inj. 9, 395-403.

13. Chan, R.C. (2005). Sustained attention in patients with mild traumatic brain injury. Clin. Rehabil. 19, 188-193.

14. Shallice, T., Stuss, D.T., Alexander, M.P., Picton, T.W., and Derkzen, D. (2008). The multiple dimensions of sustained attention. Cortex 44, 794-805.

15. Pardo, J.V., Fox, P.T., and Raichle, M.E. (1991). Localization of a human system for sustained attention by positron emission tomography. Nature 349, 61-64.

16. Gronwall, D., and Wrightson, P. (1974). Delayed recovery of intellectual function after minor head injury. Lancet 2, 605-609.

17. Gronwall, D.M. (1977). Paced auditory serial-addition task: a measure of recovery from concussion. Percept. Mot. Skills 44, 367-373.

18. Gronwall, D., and Wrightson, P. (1981). Memory and information processing capacity after closed head injury. J. Neurol. Neurosurg. Psychiatry 44, 889-895.

19. Madigan, N.K., DeLuca, J., Diamond, B.J., Tramontano, G., and Averill, A. (2000). Speed of information processing in traumatic brain injury: modality-specific factors. J. Head Trauma Rehabil. 15, 943-956.

20. Christodoulou, C., DeLuca, J., Ricker, J.H., Madigan, N.K., Bly, B.M., Lange, G., Kalnin, A.J., Liu, W.C., Steffener, J., Diamond, B.J., and $\mathrm{Ni}$, A.C. (2001). Functional magnetic resonance imaging of working memory impairment after traumatic brain injury. J. Neurol. Neurosurg. Psychiatry 71, 161-168.

21. Tombaugh, T.N. (2006). A comprehensive review of the Paced Auditory Serial Addition Test (PASAT). Arch. Clin. Neuropsychol. 21, 53-76.

22. Macciocchi, S.N., Barth, J.T., Alves, W., Rimel, R.W., and Jane, J.A. (1996). Neuropsychological functioning and recovery after mild head injury in collegiate athletes. Neurosurgery $39,510-514$.

23. Leininger, B.E., Gramling, S.E., Farrell, A.D., Kreutzer, J.S., and Peck, E.A. III. (1990). Neuropsychological deficits in symptomatic minor head injury patients after concussion and mild concussion. $\mathrm{J}$. Neurol. Neurosurg. Psychiatry 53, 293-296.

24. Lazeron, R.H., Rombouts, S.A., de Sonneville, L., Barkhof, F., and Scheltens, P. (2003). A paced visual serial addition test for fMRI. J. Neurol. Sci. 213, 29-34.

25. Lockwood, A.H., Linn, R.T., Szymanski, H., Coad, M.L., and Wack, D.S. (2004). Mapping the neural systems that mediate the Paced Auditory Serial Addition Task (PASAT). J. Int. Neuropsychol. Soc. $10,26-34$.

26. Audoin, B., Ibarrola, D., Au Duong, M.V., Pelletier, J., ConfortGouny, S., Malikova, I., Ali-Cherif, A., Cozzone, P.J., and Ranjeva, J.P. (2005). Functional MRI study of PASAT in normal subjects. MAGMA $18,96-102$

27. Cardinal, K.S., Wilson, S.M., Giesser, B.S., Drain, A.E., and Sicotte, N.L. (2008). A longitudinal fMRI study of the paced auditory serial addition task. Mult. Scler. 14, 465-471.
28. Audoin, B., Ibarrola, D., Ranjeva, J.P., Confort-Gouny, S., Malikova, I., Ali-Cherif, A., Pelletier, J., and Cozzone, P. (2003). Compensatory cortical activation observed by fMRI during a cognitive task at the earliest stage of MS. Hum. Brain Mapp. 20, 51-58.

29. Jensen, O., Gelfand, J., Kounios, J., and Lisman, J.E. (2002). Oscillations in the alpha band $(9-12 \mathrm{~Hz})$ increase with memory load during retention in a short-term memory task. Cereb. Cortex 12, 877-882.

30. Jokisch, D., and Jensen, O. (2007). Modulation of gamma and alpha activity during a working memory task engaging the dorsal or ventral stream. J. Neurosci. 27, 3244-3251.

31. Haegens, S., Cousijn, H., Wallis, G., Harrison, P.J., and Nobre, A. C. (2014). Inter- and intra-individual variability in alpha peak frequency. Neuroimage 92, 46-55.

32. Bonnefond, M., Kastner, S., and Jensen, O. (2017). Communication between brain areas based on nested oscillations. eNeuro 4. doi: 10.1523/ENEURO.0153-16.2017. eCollection 2017 Mar-Apr.

33. Tuladhar, A.M., ter Huurne, N., Schoffelen, J.M., Maris, E., Oostenveld, R., and Jensen, O. (2007). Parieto-occipital sources account for the increase in alpha activity with working memory load. Hum. Brain Mapp. 28, 785-792.

34. Haegens, S., Osipova, D., Oostenveld, R., and Jensen, O. (2010). Somatosensory working memory performance in humans depends on both engagement and disengagement of regions in a distributed network. Hum. Brain Mapp. 31, 26-35.

35. Backer, K.C., Binns, M.A., and Alain, C. (2015). Neural dynamics underlying attentional orienting to auditory representations in shortterm memory. J. Neurosci. 35, 1307-1318.

36. Long, N.M., Burke, J.F., and Kahana, M.J. (2014). Subsequent memory effect in intracranial and scalp EEG. Neuroimage 84, 488494.

37. Hanslmayr, S., Staudigl, T., and Fellner, M.C. (2012). Oscillatory power decreases and long-term memory: the information via desynchronization hypothesis. Front. Hum. Neurosci. 6, 74

38. Kondacs, A., and Szabo, M. (1999). Long-term intra-individual variability of the background EEG in normals. Clin. Neurophysiol. 110, 1708-1716.

39. Pollock, V.E., Schneider, L.S., and Lyness, S.A. (1991). Reliability of topographic quantitative EEG amplitude in healthy late-middle-aged and elderly subjects. Electroencephalogr. Clin. Neurophysiol. 79, $20-26$.

40. Napflin, M., Wildi, M., and Sarnthein, J. (2007). Test-retest reliability of resting EEG spectra validates a statistical signature of persons. Clin. Neurophysiol. 118, 2519-2524.

41. Neuper, C., Grabner, R.H., Fink, A., and Neubauer, A.C. (2005). Long-term stability and consistency of EEG event-related (de)synchronization across different cognitive tasks. Clin. Neurophysiol. $116,1681-1694$.

42. Näpflin, M., Wildi, M., and Sarnthein, J. (2008). Test-retest reliability of EEG spectra during a working memory task. Neuroimage 43, 687-693.

43. Tebano, M.T., Cameroni, M., Gallozzi, G., Loizzo, A., Palazzino, G., Pezzini, G., and Ricci, G.F. (1988). EEG spectral analysis after minor head injury in man. Electroencephalogr. Clin. Neurophysiol. $70,185-189$.

44. Bierbrauer, A.V., Weissenborn, K., Hinrichs, H., Scholz, M., and Künkel, H. (1992). [Automatic (computer assisted) EEG analysis in comparison with visual EEG analysis in patients following minor cranio-cerebral trauma (a follow-up study).] [Article in German]. EEG EMG Z Elektroenzephalogr. Elektromyogr. Verwandte Geb. $23,151-157$

45. Valdes-Hernandez, P.A., Ojeda-Gonzalez, A., Martinez-Montes, E., Lage-Castellanos, A., Virues-Alba, T., Valdes-Urrutia, L., and ValdesSosa, P.A. (2010). White matter architecture rather than cortical surface area correlates with the EEG alpha rhythm. Neuroimage 49 , 2328-2339.

46. Povlishock, J.T., and Katz, D.I. (2005). Update of neuropathology and neurological recovery after traumatic brain injury. J. Head Trauma Rehabil. 20, 76-94.

47. Kaltiainen, H., Helle, L., Liljestrom, M., Renvall, H., and Forss, N. (2018). Theta-band oscillations as an indicator of mild traumatic brain injury. Brain Topogr. 31, 1037-1046.

48. The American Congress of Rehabilitation Medicine Committee on Social, Ethical, and Environmental Aspects of Rehabilitation. (1993). Addressing the post-rehabilitation health care needs of persons with disabilities. Arch. Phys. Med. Rehabil. 74, S8-S14. 
49. Cancelliere, C., Cassidy, J.D., Cote, P., Hincapie, C.A., Hartvigsen, J., Carroll, L.J., Marras, C., Boyle, E., Kristman, V., Hung, R., Stalnacke, B.M., Rumney, P., Coronado, V., Holm, L.W., Borg, J., Nygren-de Boussard, C., Af Geijerstam, J.L., and Keightley, M. (2012). Protocol for a systematic review of prognosis after mild traumatic brain injury: an update of the WHO Collaborating Centre Task Force findings. Syst. Rev. 1, 17-4053-1-17.

50. Sherman, E.M.S., Strauss, E., and Spellacy, F. (1997). Validity of the paced auditory serial addition test (pasat) in adults referred for neuropsychological assessment after head injury. Clin. Neuropsychol. 11, 34-45.

51. Wilson, S.M., Alderman, N., Burgess, P.W., Emslie, H., and Evans, J.J. (1996). Behavioral Assessment of the Dysexecutive Syndrome. Thames Valley Test Company: Bury St. Edmund, UK,

52. Beck, A.T., Weissman, A., Lester, D., and Trexler, L. (1974). The measurement of pessimism: the hopelessness scale. J. Consult. Clin. Psychol. 42, 861-865.

53. Taulu, S., and Simola, J. (2006). Spatiotemporal signal space separation method for rejecting nearby interference in MEG measurements. Phys. Med. Biol. 51, 1759-1768.

54. Hyvarinen, A., and Oja, E. (2000). Independent component analysis: algorithms and applications. Neural Netw. 13, 411-430.

55. Hari, R., and Puce, A. (2017). MEG-EEG Primer. Oxford University Press: New York.

56. Hamalainen, M.S., and Ilmoniemi, R.J. (1994). Interpreting magnetic fields of the brain: minimum norm estimates. Med. Biol. Eng. Comput. 32, 35-42.

57. Liljestrom, M., Kujala, J., Jensen, O., and Salmelin, R. (2005). Neuromagnetic localization of rhythmic activity in the human brain: a comparison of three methods. Neuroimage 25, 734-745.

58. Gross, J., Kujala, J., Hämäläinen, M., Timmermann, L., Schnitzler, A., and Salmelin, R. (2001). Dynamic imaging of coherent sources: studying neural interactions in the human brain. Proc. Natl. Acad. Sci. U. S. A. 98, 694-699.

59. Van Veen, B.D., van Drongelen, W., Yuchtman, M., and Suzuki, A. (1997). Localization of brain electrical activity via linearly constrained minimum variance spatial filtering. IEEE Trans. Biomed. Eng. 44, 867-880.

60. Gramfort, A., Luessi, M., Larson, E., Engemann, D.A., Strohmeier, D., Brodbeck, C., Goj, R., Jas, M., Brooks, T., Parkkonen, L., and Hamalainen, M. (2013). MEG and EEG data analysis with MNEPython. Front. Neurosci. 7, 267.

61. Gramfort, A., Luessi, M., Larson, E., Engemann, D.A., Strohmeier, D., Brodbeck, C., Parkkonen, L., and Hamalainen, M.S. (2014). MNE software for processing MEG and EEG data. Neuroimage 86, 446460

62. van Vliet, M., Liljeström, M., Aro, S., Salmelin, R., and Kujala, J. (2018). Analysis of functional connectivity and oscillatory power using DICS: from raw MEG data to group-level statistics in Python. Front. Neurosci. 12, 586

63. Tallon-Baudry, C., Bertrand, O., Delpuech, C., and Pernier, J. (1997). Oscillatory $\gamma$-band $(30-70 \mathrm{~Hz})$ activity induced by a visual search task in humans. J. Neurosci. 17, 722-734.

64. Fischl, B., Salat, D.H., Busa, E., Albert, M., Dieterich, M., Haselgrove, C., van der Kouwe, A., Killiany, R., Kennedy, D., Klaveness, S., Montillo, A., Makris, N., Rosen, B., and Dale, A.M. (2002). Whole brain segmentation: automated labeling of neuroanatomical structures in the human brain. Neuron 33, 341-355.

65. Desikan, R.S., Segonne, F., Fischl, B., Quinn, B.T., Dickerson, B.C., Blacker, D., Buckner, R.L., Dale, A.M., Maguire, R.P., Hyman, B.T., Albert, M.S., and Killiany, R.J. (2006). An automated labeling system for subdividing the human cerebral cortex on MRI scans into gyral based regions of interest. Neuroimage 31, 968-980.

66. Maurer, U., Brem, S., Liechti, M., Maurizio, S., Michels, L., and Brandeis, D. (2015). Frontal midline theta reflects individual task performance in a working memory task. Brain Topogr. 28, 127-134.

67. Gutmann, B., Mierau, A., Hülsdünker, T., Hildebrand, C., Przyklenk, A., Hollmann, W., and Strüder, H.K. (2015). Effects of physical exercise on individual resting state EEG alpha peak frequency. Neural Plast 2015, 717312.

68. Mierau, A., Klimesch, W., and Lefebvre, J. (2017). State-dependent alpha peak frequency shifts: experimental evidence, potential mechanisms and functional implications. Neuroscience 360, 146-154.

69. Saggar, M., King, B., Zanesco, A., MacLean, K., Aichele, S., Jacobs, T., Bridwell, D., Shaver, P., Rosenberg, E., Sahdra, B., Ferrer, E., Tang, A., Mangun, G., Wallace, B.A., Miikkulainen, R., and Saron, C. (2012). Intensive training induces longitudinal changes in meditation state-related EEG oscillatory activity. Front. Hum. Neurosci. 6, 256.

70. Kostyunina, M.B., and Kulikov, M.A. (1996). Frequency characteristics of EEG spectra in the emotions. Neurosci. Behav. Physiol. 26, 340-343.

71. Rogers, J.M., Donnelly, J., and Wilson, P.H. (2015). Source localization of an event-related potential marker of executive attention following mild traumatic brain injury. Neuroreport 26, 903-907.

72. Delazer, M., and Benke, T. (1997). Arithmetic facts without meaning. Cortex 33, 697-710.

73. van Harskamp, N.J., and Cipolotti, L. (2001). Selective impairments for addition, subtraction and multiplication. implications for the organisation of arithmetical facts. Cortex 37, 363-388.

74. Göbel, S.M., Rushworth, M.F., and Walsh, V. (2006). Inferior parietal RTMS affects performance in an addition task. Cortex 42, 774-781.

75. Owen, A.M., McMillan, K.M., Laird, A.R., and Bullmore, E. (2005) N-back working memory paradigm: a meta-analysis of normative functional neuroimaging studies. Hum. Brain Mapp. 25, 46-59.

76. Szczepanski, S.M., and Knight, R.T. (2014). Insights into human behavior from lesions to the prefrontal cortex. Neuron 83, 1002-1018.

77. Barbey, A.K., Koenigs, M., and Grafman, J. (2012). Dorsolateral prefrontal contributions to human working memory. Cortex 49, 11951205.

78. Hanslmayr, S., and Staudigl, T. (2014). How brain oscillations form memories â€" A processing based perspective on oscillatory subsequent memory effects. Neuroimage 85, 648-655.

79. Arico, P., Borghini, G., Di Flumeri, G., Colosimo, A., Graziani, I., Imbert, J.P., Granger, G., Benhacene, R., Terenzi, M., Pozzi, S., and Babiloni, F. (2015). Reliability over time of EEG-based mental workload evaluation during Air Traffic Management (ATM) tasks. Conf. Proc. IEEE Eng. Med. Biol. Soc. 2015, 7242-7245.

Address correspondence to:

Hanna Kaltiainen, MD

Department of Neuroscience and Biomedical Engineering

School of Science

Aalto University

PO Box 12200

FI-00076 AALTO

Espoo

Finland

E-mail: hanna-leena.kaltiainen@aalto.fi 